\title{
Happy Birthday, Anästhesie!
}

Herausgeber

G. Geldner, Ludwigsburg

T. Hachenberg, Magdeburg

W. Koppert, Hannover

C. Krier, Stuttgart

G. Marx, Aachen

N. Roewer, Würzburg

J. Scholz, Kiel

C. Spies, Berlin

H. Van Aken, Münster

H. Wulf, Marburg

K. Zacharowski, Frankfurt/Main
Haben Sie auch gefeiert vergangene Woche? Am 16. Oktober wurde Ihr Fachgebiet stolze 167 Jahre alt. Vor exakt so vielen Jahren nahm der Zahnarzt William Thomas Green Morton im Massachusetts General Hospital in Boston die erste öffentliche Äthernarkose vor. Das Ereignis gilt als offizielle Geburtsstunde der modernen Anästhesie und wird heute als „Ether Day“ auf der ganzen Welt gefeiert. Anlässlich hierzu erinnern Goerig und Wulf in ihrem Artikel ab o S. 648 an einige bekannte und weniger bekannte Geschehnisse rund um diesen geschichtsträchtigen Tag und die Einführung der modernen Anästhesie.

Seit 2012 wird der Ether Day auch in Deutschland offiziell gefeiert. Vergangenes Jahr riefen der Berufsverband Deutscher Anästhesisten (BDA) und die Deutsche Gesellschaft für Anästhesiologie und Intensivmedizin (DGAI) zum 1. Weltanästhesie-Tag auf. Anästhesisten aus über 200 Kliniken folgten dem Aufruf und veranstalteten Aktionstage, Infoveranstaltungen oder Telefonaktionen, um die Bevölkerung auf ihr Fachgebiet aufmerksam zu machen und sie zu informieren.
Das große Engagement wurde belohnt und das Projekt „Weltanästhesie-Tag 2012“ mit dem „Health Media Award 2013“ in der Rubrik „Patientenkommunikation“ ausgezeichnet.

Auch vergangene Woche haben wieder hunderte von Anästhesisten mit unterschiedlichen Veranstaltungen für ihr Fachgebiet geworben. Vielleicht waren ja auch Sie dieses Mal dabei und haben sich mit einer ganz besonderen Aktion am Weltanästhesie-Tag beteiligt?

Dann bewerben Sie sich für den SELMA-Award! Mit diesem erstmalig verliehenen Kommunikationspreis im Fachgebiet Anästhesiologie möchten der BDA und die DGAI gelungenen Projekten eine würdige Plattform geben. Teilnehmen können alle Fachärzte für Anästhesiologie in Deutschland, die sich mit einem Projekt am Weltanästhesie-Tag 2013 beteiligt haben. Nähere Informationen hierzu finden Sie im Internet unter o www.weltanaesthesietag.de

\section{Mit herzlichen Grüßen}

Ihre Herausgeber und Ihre Redaktion
Experten-Panel

B. Bein, Kiel

E. Biermann, Nürnberg

J. Biscoping, Karlsruhe

B. Böttiger, Köln

M. Bucher, Halle

H. Bürkle, Freiburg

B. Dirk s, Ulm

V. von Dossow, München

L. Eberhart, Marburg

U. Ebmeyer, Magdeburg

M. Fischer, Göppingen

W. Gogarten, München

J. Graf, Frankfurt/Main

S. Grond, Detmold

U. Kaisers, Leipzig

C. Kill, Marburg

U. Klein, Nordhausen

S. Kozek-Langenecker, Wien

P. Kranke, Würzburg

L. Lampl, Ulm

J. Martin, Göppingen

A. Meißner, Soest

C. Nau, Erlangen

J. Pfefferkorn, Stuttgart

P. Rosenberger, Tübingen

M. Schäfer, Berlin

T. Schnider, St. Gallen

T. Schürholz, Aachen

U. Schwemmer, Neumarkt

T. Standl, Solingen

F. Stüber, Bern

R. Sümpelmann, Hannover

M. Tramèr, Genf

K. Ulsenheimer, München

T. Volk, Homburg/Saar

A. Walther, Stuttgart

F. Wappler, Köln

E. Weis, Nürnberg

Organschaften

Deutsche Gesellschaft für Anästhesiologie und Intensivmedizin

Österreichische Gesellschaft für Anaesthesiologie, Reanimation und Intensivmedizin

AINS indexiert in Medline, Embase, Scopus Science Citation Index Expanded

Verlag

www.thieme.de/ains

www.thieme-connect.de/ejournals

wrw.thieme-connect.de/ejournals

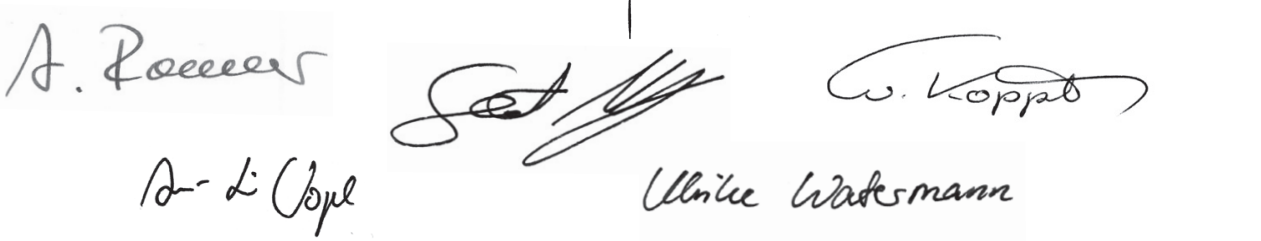

